Who could benefit most from treatment of acute pulmonary embolism with rivaroxaban? Commentary to the article: "Acute pulmonary embolism treatment with rivaroxaban results in a shorter duration of hospitalisation compared to standard therapy: an academic centre experience"

published in "Kardiologia Polska" 2016; 74, 7: 650-656

\title{
Anetta Undas
}

Institute of Cardiology, Jagiellonian University Medical College and Centre for Research and Medical Technology, John Paul II Hospital, Krakow, Poland

Meta-analyses of phase III trials have compellingly demonstrated that non-vitamin $\mathrm{K}$ oral anticoagulants (NOACs), including rivaroxaban and apixaban alone and dabigatran as well as edoxaban, after parenteral anticoagulant induction, are as effective as, and probably safer than, standard treatment with heparin/warfarin of acute venous thromboembolism (VTE). The relative efficacy and safety of the NOACs seem consistent across a wide range of patients [1]. Moreover, the NOACs significantly simplify VTE treatment because they are administered at fixed doses without any dietary restrictions along with limited interactions with other drugs, and, notably, no routine anticoagulation monitoring is required [2]. Despite growing evidence for the favourable risk-benefit profile of NOACs compared to warfarin, few studies have assessed the impact of NOACs used for treatment of acute VTE in real life. The present study by Paczyńska et al. [3] could be of particular interest for Polish cardiologists by providing such data from a well-known Warsaw centre following an investigation involving 215 consecutive acute pulmonary embolism (PE) patients hospitalised in the years 2013-2014. The study shows that patients treated with rivaroxaban (following a maximum of three days' heparin administration) compared with those receiving vitamin $\mathrm{K}$ antagonists (VKA) stayed in hospital for a shorter period of time both when they had simplified PE severity index $(\mathrm{SPESI})=0$ and $\mathrm{SPESI} \geq 1$ [3]. To explain this observation, comparative analysis of demographic and clinical characteristics for the PE patients on rivaroxaban and those on the standard therapy is needed. One might expect that the choice of rivaroxaban over the standard treatment in this cohort is driven by lower age of patients, fewer comorbidities, absence of dual antiplatelet agents, and normal renal function. Danish nationwide data from 2011 to 2013 published recently documented the pattern of use of currently available NOAC and warfarin in patients with atrial fibrillation (AF) and demonstrated that for this indication, NOAC are more commonly used in female subjects at older age and in those with prior stroke, and less commonly in subjects with a history of chronic kidney disease, myocardial infarction, and heart failure [4]. Even if the current cohort was younger (median, 65 years) than most AF populations treated with NOACs [2], but higher than the average age of patients in each clinical trial on NOACs in VTE (55-60 years) [5], most associations reported in AF could be applicable to the VTE patients, although prolonged use of low-molecular-weight heparins, not only when strongly indicated, i.e. in cancer- or pregnancy-associated PE, is relatively common in Poland. From my experience, in 2015 NOACs are preferred over warfarin in patients of both sexes with low- and intermediate-risk acute PE at ages below 65 years in whom renal function is normal or slightly reduced and a history of gastrointestinal bleeding is negative (A. Undas, unpublished data). It would be extremely interesting to show which factors influenced the Warsaw doctors' decisions to use rivaroxaban or the standard therapy, apart from high-risk PE in which preferentially heparins followed by VKA antivitamin $\mathrm{K}$ were most likely to be used. This issue is unclear after having read the paper by Paczyńska et al. [3]. Another issue that deserves comment is a temporal prescription pattern of rivaroxaban compared with warfarin or acenocoumarol throughout the two-year study period.

Current European Society of Cardiology guidelines on acute PE management do not endorse NOACs over warfarin. There is a consensus that the choice of anticoagulation regimen should be personalised based on the relative efficacy and safety of different agents across subgroups stratified by thrombotic and bleeding risk, as well as on other clinical factors, e.g. drug interactions or compliance. I encourage the authors to continue their study and extend follow-up to provide much-needed data on the outcomes of rivaroxaban versus standard therapy of PE in Poland. To optimise treatment for acute PE in the real-world setting, further research 
is warranted to identify PE patients who may preferentially be managed with rivaroxaban or other NOACs in order to ease the burden of this potentially lethal disease.

Conflict of interest: Honoraria for lectures received from Bayer, a manufacturer of Xarelto (rivaroxaban).

\section{References}

1. van der Hulle T, Kooiman J, den Exter PL et al. Effectiveness and safety of novel oral anticoagulants as compared with vitamin $\mathrm{K}$ antagonists in the treatment of acute symptomatic venous thromboembolism: a systematic review and meta-analysis. J Thromb Haemost, 2014; 12: 320-328.
2. Undas A, Pasierski T, Windyga J, Crowther M. Practical aspects of new oral anticoagulant use in atrial fibrillation. Pol Arch Med Wewn, 2014; 124: 124-135.

3. Paczyńska M, Kurnicka K, Lichodziejewska B et al. Acute pulmonary embolism treatment with rivaroxaban results in a shorter duration of hospitalisation compared to standard therapy: an academic centre experience. Kardiol Pol, 2016; 74: 650-656. doi: 10.5603/KP.a2015.0253.

4. Olesen JB, Sørensen R, Hansen ML et al. Non-vitamin K antagonist oral anticoagulation agents in anticoagulant naïve atrial fibrillation patients: Danish nationwide descriptive data 2011-2013. Europace, 2015; 17: 187-193.

5. Bacchus F, Schulman S. Clinical experience with the new oral anticoagulants for treatment of venous thromboembolism. Arterioscler Thromb Vasc Biol, 2015; 35: 513-519. 